as used for electrodes in the bath, a series of metal handles of different sizes, covered with house flannel, have been employed. (Fig. 7.) The hands have gradually opened out. Small handles are used at first, and gradually increased in size as the patient's hand is able to receive them. The handles are attached to the negative pole of the battery instead of the foot plate. The current then enters the body at the spine, and leaves it by the hand which grasps the handle, and is thus more localised. But this local application of the current should only be used for about the last five minutes of the bath; it should never altogether take the place of the general bath, which probably influences the

FIG. 7.
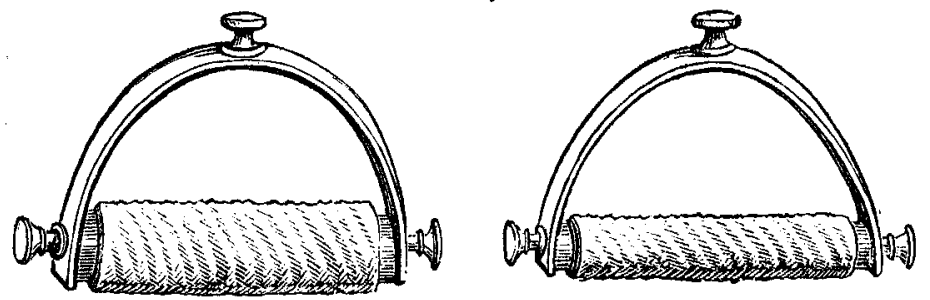

whole system. The hands may also be made to grasp a metal bar supported across the bath and covered with house flannel, ${ }^{1}$ and attached to one pole of the battery. In this position the hands are raised out of the water and probably get more of the current, but the entire current flowing through the battery must be very greatly reduced. When concentrated in this manner on the hands the patient cannot bear more than ten or twelve nilliamperes. When any of these changes in the direction of the current are made the strength has to be previously reduced to zero, and then increased again after the change has been made. If the wire were detached from the plate at the bottom of the bath when the full strength of 200 milliampères was passing, the patient would receive a very unpleasant and severe shock.

On looking back after the completion of a course of baths to the condition of the patient before the treatment was commenced, it is generally possible to notice some improvement. A patient, for instance, who could only rise from one step to the next, on going upstairs, by pulling on the balustrade, can make the ascent without such assistance; or a woman who could not hold her needle finds that she is able to use it slowly. The theory that the origin of chronic rheumatoid arthritis in many women may be through reflex action consequent upon the irritation of the spinal cord by prolonged congestion and inflammation of the pelvic organs is somewhat strengthened by the fact that the sedative action of the positive pole on the spinal marrow appears to be followed by some alleviation of the symptoms. The chances of this theory being probably a correct one led to the adoption of the use of the positive pole at the head of the bath. The rheumatoid condition is frequently associated with catarrhal endometritis. The question arises as to the relation they bear to each other as cause and effect. The superiority of the results claimed for the treatment of these pelvic troubles by electricity, in which the negative pole is applied to the uterus and the positive to the lower dorsal vertebræ, may also partly be due to the sedative action of the positive pole on the spinal cord.

Rheumatic arthritis of hip., knee-, or shoulder-joints.When the larger joints are affected with rheumatic arthritis, such as the keees, hips, or shoulders, the current can be localised by the use of the smaller metal plates (Fig. 2, p. 710), one being placed on either side of the bath, so as to include the affected joints between them. The joints should be afterwards subjected to rubbing and passive movements. Local arm baths or foot baths are sometimes given when one limb or joint only is affected. A porcelain or small wooden bath is the best to employ, and two small metal plates attached to the respective poles of a battery are placed one on either side of the affected member and a current allowed to pass. Sometimes one electrode only is in the water; the other-a carbon disc electrode attached to the negative pole-being applied to the affected joint or limb; for instance, to a rheumatic knee or to the arm in a case of cervico-brachial neuralgia. But ordinary galvanising with. out the intervention of a bath seems to be more suitable for these localised affections, and does not occupy nearly so long a time or so exhaust the battery.
Gonorrhoral rheumatism. - This affection has also been treated by electric baths on the same plan as that followed in the treatment of rheumatoid arthritis, but not with quite such satisfactory results. The positive pole is placed at the head of the bath, on the supposition that urethral irritation reflected on to the spinal cord may be one of the factors influencing the incidence of the disease. It is advisable to employ massage for the affected limbs after the bath.

Gont.-The galvanic bath is said to be efficacious in gout It helps to effect that much-to-be-desired metabolisis mentioned by Professor Latham-viz., the conversion of uric acid into urea. In a recent paper on the formation of uric acid Dr. Latham has sought to prove that the presence of uric acid in the blood is due to the imperfect metabolism of glycocine, which takes place under certain conditions, one being an insufficient amount of exercise. When a proper amount of exercise is taken the glycocine is trans. formed into urea and normally eliminated by the kidneys. He has also sought to prove that this more-to.bedesired metabolisis is dependent upon a due amount of nerve force, and that the production of nerve force is encouraged by exercise. It has also been proved that the contraction of muscle produces electrical currents. Urea is due to the oxidation of uric acid, and Professor Dewar of Cambridge has produced urea from urie acid by making and breaking a current of electricity when passing through it Professor Dewar has also experimented upon the electrolysis of urea. In referring to this experiment Dr. Latham makes the interesting remark that "if there be any correspondence at all between nerve force and the electrical current this experiment possesses great significance." From the beneficial effects of the treatment of gout by the electric bath these surmises and observations receive support. The tophi in gout are composed of urate of soda, and are removed by galvanism, most likely by a process akin to electrolysis, the urate being split up at the negative pole.

Lehr says that in gout the electric bath is a palliative of great value.

(To be continued.)

\section{THE ANILINE DYES AS ANTISEPTICS.}

\section{BY J. STILLING, M.D.,}

PROFESSOR IN THE UNIYERSITY OF STRASSBURG

Translated and much condensed

By EDGAR STEVENSON, M. B. ABERDEEN.

I MUST begin my second article by touching on some points that have been suggested to me in the various communications I have received on this subject. It has been said that irritation follows the application of methyl violet. I have nyself never seen irritation follow the application of a 1 in 1000 solution of methyl violet in animals, or in men, though I have treated hundreds of patients with it. I have even used 1 per cent. solutions with only a little hyperæmia resulting, which quickly disappeared on diminishing the dose. This has been confirmed by Drs. Wanscher, Nagues, and others. In the course of my experiments I have made large skin wounds in the backs of rabbits, and by loosening the connective tissue have produced a cavity which I have filled with large quantities (two or three grammes) of the pure substance. These large wounds have healed without the slightest swelling or inflammation in the surrounding tissues, and the general condition of the animals remained unimpaired. For external application in all surgical cases the substance can be regarded as quite non-poisonous. A gramme of an aniline dye is, however, on account of its small weight, so large a quantity that it suffices to treat wound or ulcer of considerable size. Graefe, in the Fort schritte der Medicin, June, 1890, says that he has found methyl violet to have a local inflammatory action, so much so that a subcutaneous injection of $0.3 \mathrm{cc}$. of a 2 in 1000 solution in a rabbit's ear has caused an obstinate inflammation. I can only say that this is entirely contrary to all my results, and, as I have already stated in my first article, I have injected large quantities of the dye into the peritoneal carity of animals without the least sign of inflammation. These

\footnotetext{
2 Erb's Electrotherapeutics, translated by De Watterille, p. 281.
} 
results of mine have been fally confirmed by the investigations of Wanscher, Bresgen, and others. ('rraefe's results are probably due to impurities in the dye, such as chloride of zinc, arsenic, and copper sulphate. The results of my experiments briefly stated are as follows:-1. Methyl violet is about three times as strong as sublimate in its action on the anthrax bacillus, and quite as effectual as sublimate in its action on the staphylococcus pyogenes aureus. 2 . It is a perfectly non-poisonous substance. 3 . In consequence of this, it is immaterial how strong the solutions may be, even up to the use of the pure substance itself. 4. It does not coagulate egg albumen. 5. It possesses an extraordinary power of diffusion, penetrating in the eye like atropin.

In its action on the normal and pathological states of the cornea the following may be noticed: The normal cornea is only stained if it be scratched and the substance immediately applied, whilst the diseased cornea is very quickly and deeply stained. The more recent the corneitis, the longer the stain lasts, and the larger the quantity of the dye deposited from the solution; the staining can then be observed to gradually fade after a few minutes, and this warrants a favourable prognosis, the opposite to this being an unfavourable sign. Granulations of the conjunctiva are also easily and deeply stained, whilst the normal conjunctiva, in spite of the superficial layer of cast-off cells. is by no means so deeply stained as the granulations. Blepharitis is quickly cured by the application of a 2 per cent. ointment or of a pencil to the roots of the hairs. It is better in these cases to use the yellow dye-auramin, -on account of the slight stain produced by it. Since I hegan to use the aniline treatment I have generally cured jad cases of blepharitis in a few days.

As regards conjunctivitis I have had the very best results in even the worst forms of the disease by using drops of a 1 in 1000 solution of methyl violet, or by sprinkling with a 2 per cent. yellow or blue powder. This treatment will practically cure a case in twenty-four hours. I have also had good results in blennorrhœea, croupous conjunctivitis, and iritis serosa ; for keratitis parenchymatosa the methyl violet solution is almost a specific. Dr. Wanscher of Copenhagen has obtained excellent results in blennorrbcal and gonor. rhoeal ophthalmia by pencilling the conjunctiva several times a day till the tissue was quite blue. In cases of ordinary acute, subacute, and chronic conjunctivitis I have so far obtained better results with auramin solution of 1 in 1000 strength than with the other substances. The patients also prefer it, as they invariably say that it has a very pleasant, cooling action. Very severe cases of granular lids are not often seen here, but in the milder cases I find the application of the pencil to the granulations quite sufficient. With this treatment there are the advantages that the application is not painful, as is the case with nitrate of silver or sul. phate of copper, and also that a cure is more speedily effected.

I might here mention that my friend, Professor Goltz, informs me that purulent inflammation of the cornea going on to perforation and destruction of the eye frequently occurs in dogs after operations on the cerebrum. This inflammation he has succeeded in curing in a few days by at once applying the 1 in 1000 methyl violet solu tion. As regards the use of methyl violet in ordinary surgical practice, I have received communications from surgeons from all parts of the Continent, saying that they have been most successful in the aniline treatment of ulcers, abscesses, burns, \&c., and also of wounds, all suppuration being prevented. The substance must be used till the tissues are deeply stained. I quote one such communication from Dr. Voigt. ${ }^{1}$ He says that " the very best results have followed the use of the pure powder, which is easily applied with a brush, especially in acute suppuration, such as carbuncle, whitlow, phlegmon, \&c., the pain and suppuration being simultaneously stopped. One need not, if it be necessary, hesitate to make deep incisions in order to bring the substance into thorough contact with the diseased parts. The blue zone can be traced for $\frac{1}{2}$ to $2 \mathrm{~mm}$. deep in the tissues.

The following are the preparations of the dyes:-

1. Pure methyl violet. - To be used as powder for large wounds and ulcers,

2. Large pencils.-For small wounds, burns, \&c. For purulent cases the blue pencil is better than the yellow, on account of its greater antiseptic property.

1 Aerztl. Centr. Anz., 1890, No. 25.
3. Small pencils.-For application to the eye, in cases of corneal ulcer \&c.

4. Powders. - Of 1 in 1000 strength for mild cases of conjunctivitis, and for more severe cases (blennorrhoea) of 2 per cent. strength. These can also be used as a snuff in affections of the nasal mucous membrane.

5. Ointments. - In strength varying from 2 per cent. to 1 in 10.

6. Solutions.-Used in strengths of 1 in 1000 to 1 per cent. The 1 in 1000 solution is to be used for ordinary cases of conjunctivitis, keratitis, \&c, and in non-purulent cases the yellow dye auramin may be used, on account of its abovementioned cooling properties. The solutions should be filtered and kept in dark glass bottles, and changed every eight days.

In conclusion, I must once more call attention to the necessity of the substances used being absolutely pure.

Strassburg.

\section{THE BULBAR CRISES OF DIPHTHERITIC}

\section{PARALYSIS OCCURRING IN CHILDREN.}

BY L. G. GUTHRIE, M B. Oxon., M.R.C.P. LoND., ASSISTANT PHYSICIAN, NORTH-WEST LONDON HOSPITAL; PHYSICIAN, REGENT'S-PARK HOSPITAL FOR EPILEPSY AND PARALYSIS ; PATHOLOGIST (LATE RFGISTRAR), PADDINGTON-GREEN CHILDREN'S HOSPITAL.

THE object of this paper is to describe symptoms of diph. theritic paralysis which are often unexpected, which may often be unrecognised, and which often prove fatal. The symptoms to which I refer may be described as "bulbar crises." They consist in sudden and severe functional disturbances of the vital medullary centres. They resemble in some respects the symptoms of so.called acute bulbar palsy, and it is possible that some cases of the latter disease may be of unsus pected diphtheritic origin. ${ }^{1}$ I shall presently describe them fully; but, first, as to the conditions under which they occur.

The premonitory symptoms of bulbar crises are as follows. Children are usually seen in the second or third week of diphtheritic paralysis. They are then suffering from slight weakness of the legs and loss of knee.jerks, staggering gait, a nasal voice, occasional regurgitation of fluids through the nose, and sometimes a "squint." The common reason for bringing them is that they "fall about," seem weak, and "want strengthening medicine." The other symptoms are seldom elicited except on cross-examination. Sometimes, however, children are brought because their speech has. lately become defective. The initial sore throat is seldom mentioned, sometimes it is denied. ${ }^{2}$ They are often well nourished and healthy looking. They take food well, except for the occasional return of fluids; they sleep well, and. there may seem little cause for anxiety as to their condition. Yet a closer examination will often give grounds for alarm. There is a peculiar apathy and lethargy about the patient which is very characteristic. He will lie all day on his back without attempting to move. He will smile placidly when spoken to, but will not answer unless pressed to do so. Then, speech is attended by obvious effort. A deep, sudden inspiration is taken. The voice escapes in sudden expiratory gasps ; it is not only nasal, but also extremely weak and hoarse. The weakness and hoarseness suggest incomplete closure of the vocal cords (adductor paralysis); so also does the occasional loose and almost noiseless cough. More probably, however, there is a certain amount of bilateral paresis of the cords. ${ }^{3}$ The character of the respirations often affords indications ox danger. They are not usually hurried. They average about 20 per minute, but inspiration is sudden, deep, and forcible whilst expiration is short and weak. At intervals there is a long-drawn sigh. This sign is almost invariably present, and is highly significant of an approaching crisis. At

If. Dr. Stretch Dowse, Merl. Soc. Trans., Jan. 29th, 1877: “Extreme voraciousness distinguishes ordinary bulbar paralysis from the bulbar paralysis of diphtheria.

2 It may be objected that in these cases there is no evidence that the disease is diphtheritic. A similar objection might be raised to the diagnosis of well-marked tertiary syphilis, where no history of chancre can be obtained.

'The inspiratory stridor so common after tracheotomy is seldom noticed, except when this operation has been performed. 